Physics Vo1. 2, No. 2, pp. 81-94, 1965. Physics Publishing Co. Printed in Great Britain.

\title{
MISE EN EVIDENCE PAR RESONANCE MAGNetique deS PROPRIETES D' UNE BANDE D' IMPURETES DANS LE SILICIUM
}

\author{
D. JEROME, CH. RYTER et J.M. WINTER \\ Service de Physique du Solide et de Résonance Magnétique \\ Centre d'Etudes Nucléaires de Saclay, France \\ (Received 14 April 1965)
}

\begin{abstract}
Some consequences of the occurrence of a narrow impurity band in heavily doped semi-conductors have been studied at low temperature by magnetic resonance. The experiments have been done on phosphorus-doped silicon $\left(2,510^{18} \mathrm{P} / \mathrm{cm}^{3}\right)$. The nuclear relaxation of $\mathrm{Si}^{29}$ nuclei near the impurity centers is due to contact hyperfine interaction; it is obtained that the electron density shows a very large maximum near the impurities, although remaining at a very low value between the impurities.
\end{abstract}

The relaxation and dynamic polarisation by Overhauser effect of $\mathrm{Si}^{29}$ far from impurity centers is explained by a spin diffusion process.

The impurity band width $\left(\sim 19^{\circ} \mathrm{K}\right)$ is deduced from the measurement of the paramagnetic part of the electron susceptibility at low temperature.

\section{Introduction}

DANS les semiconducteurs extrinsèques, la résistivité électrique à basse température diminue très rapidement lorsque la concentration des impuretés atteint et dépasse une valeur critique $N_{c}[1,2,3,4]$. Mott $[5,6]$ a interprété ce phénomène comme une transition vers l'état métallique $[7,8]$ et 1 ' on constate alors:

- que la conductivité à basse température devient indépendante de la température, et notamment tend vers une valeur finie à température nulle.

- que la constante de Hall devient indépendante de la température, ce qui indique que le nombre de porteurs libres est égal au nombre de centres d'impuretés [9]. $[10]$.

- que la susceptibilité magnétique est celle qui correspond à un gaz d’électrons dégénérés

C' est aussi à partir de la concentration critique $N_{c}$ que le concept de bande devient valable; cette concentration est déterminée par la relation

$$
0.62 / N_{c}^{1 / 3} b^{*}=0.3
$$


où $b^{*}$ est le rayon d' orbite de Bohr [9]. Dans l'approximation hydrogénoide, $b^{*}$ est déterminé à partir de l'énergie d'ionisation observée. Pour le phosphore dans le silicium, on $a b^{*}=a^{*}$ $\left(E_{\text {uasse eff }} / E_{\mathrm{obs}}\right)^{1 / 2}[26]$, soit $b^{*}=16.3 \AA$ lorsque $a^{*}=21 \AA$ [14]. La concentration calculée à l'aide de (1) est: $N_{c}=2 \times 10^{18} \mathrm{P} / \mathrm{cm}^{3}$.

Pour l'échantillon de silicium dopé au phosphore sur lequel porte ce travail, $N=2.5 \times 10^{18}$ $P / \mathrm{cm}^{3}$. Cette concentration est déduite de la résistivité $\rho=0.014 \Omega . \mathrm{cm}$ à la température ordinaire. * ${ }^{\dagger}$

A la température de l'hélium liquide, le spectre de résonance électronique des impuretés de phosphore en quantité suffisamment faible dans du silicium présente des raies séparées [14] dues à des atomes isolés, et éventuellement d'autres raies dues à des amas de plusieurs impuretés dont les spins électroniques sont couplés par une interaction d'échange [15]. Pour des concentrations croissantes, le recouvrement des fonctions d'onde augmente, les électrons tendent à se délocaliser [14], et, dans la région de concentration qui nous intéresse, le spectre de résonance électronique se réduit à une raie unique, étroite, homogène, caractérisée par un facteur $g=1.99875 \pm 0.00010$ et un temps de relaxation de $1^{\prime}$ ordre de $10^{-6} \mathrm{~s}$, à la température de $4.2^{\circ} \mathrm{K}$ et à des températures inférieures. Elle a les apparences d' une raie de résonance d' électrons de conduction dans un métal.

Une sérieuse complication dans l'interprétation provient de la distribution au hasard des centres donneurs qui empêche de calculer simplement une structure de bande en partant de fonctions d'onde d'impuretés isolées $[11,12,13]$.

Toutefois, les résultats des expériences s' expliquent bien en admettant que la fonction d'onde des électrons présente des maximums très importants à l'emplacement des atomes de phosphore et, au contraire, des intensités faibles mais non nulles dans les régions intermédiaires. Les noyaux de ${ }^{2}{ }^{9} \mathrm{Si}$ ( $d^{\prime}$ abondance $4.7 \%$ ) peuvent alors être rangés en deux groupes aux propriétés complètement différentes:

- Les noyaux proches d' un atome de phosphore, individuellement couplés aux électrons, sont soumis à des interactions hyperfines très différentes les unes des autres, et ne peuvent pas se mettre en équilibre thermique entre eux.

- Les noyaux éloignés d' un atome de phosphore pour lesquels le concept de température de spin s'applique; les échanges d'énergie avec le réseau se font alors par diffusion de spin. Il existe une barrière de diffusion produite par le voisinage d' un atome de phosphore.

\section{Polarisation Dynamique}

La polarisation dynamique fournit d'importantes précisions sur la nature de l'hamiltonien responsable de la relaxation. L' augmentation extrême $A_{\mathbf{m a x}}$ de l'aimantation nucléaire que produit l' effet Overhauser est donnée par $-\gamma_{e} / \gamma_{n}$ pour un couplage scalaire et $+(1 / 2) \gamma_{e} / \gamma_{n}$ pour un couplage dipolaire entre le noyau considéré et un centre paramagnétique [16], et l'on $a$, pour une interaction scalaire,

- D'aprés les mesures de J.C. Irvin, 1960, Communication particulière.

$\dagger$ Echantillon de silicium fourni par Merck. 
où $b^{*}$ est le rayon d' orbite de Bohr [9]. Dans $1^{\prime}$ approximation hydrogénoide, $b^{*}$ est déterminé à partir de l'énergie d'ionisation observée. Pour le phosphore dans le silicium, on $a b^{*}=a^{*}$ $\left(E_{\text {uasse eff }} / E_{\mathrm{obs}}\right)^{1 / 2}[26]$, soit $b^{*}=16.3 \AA$ lorsque $a^{*}=21 \AA$ [14]. La concentration calculée à l'aide de (1) est: $N_{c}=2 \times 10^{18} \mathrm{P} / \mathrm{cm}^{3}$.

Pour l'échantillon de silicium dopé au phosphore sur lequel porte ce travail, $N=2.5 \times 10^{18}$ $P / \mathrm{cm}^{3}$. Cette concentration est déduite de la résistivité $\rho=0.014 \Omega . \mathrm{cm}$ à la température ordinaire. * ${ }^{\dagger}$

A la température de l'hélium liquide, le spectre de résonance électronique des impuretés de phosphore en quantité suffisamment faible dans du silicium présente des raies séparées [14] dues à des atomes isolés, et éventuellement d'autres raies dues à des amas de plusieurs impuretés dont les spins électroniques sont couplés par une interaction d'échange [15]. Pour des concentrations croissantes, le recouvrement des fonctions d'onde augmente, les électrons tendent à se délocaliser [14], et, dans la région de concentration qui nous intéresse, le spectre de résonance électronique se réduit à une raie unique, étroite, homogène, caractérisée par un facteur $g=1.99875 \pm 0.00010$ et un temps de relaxation de $1^{\prime}$ ordre de $10^{-6} \mathrm{~s}$, à la température de $4.2^{\circ} \mathrm{K}$ et à des températures inférieures. Elle a les apparences d' une raie de résonance d' électrons de conduction dans un métal.

Une sérieuse complication dans l'interprétation provient de la distribution au hasard des centres donneurs qui empêche de calculer simplement une structure de bande en partant de fonctions d'onde d'impuretés isolées $[11,12,13]$.

Toutefois, les résultats des expériences s' expliquent bien en admettant que la fonction d'onde des électrons présente des maximums très importants à l'emplacement des atomes de phosphore et, au contraire, des intensités faibles mais non nulles dans les régions intermédiaires. Les noyaux de ${ }^{2}{ }^{9} \mathrm{Si}$ ( $d^{\prime}$ abondance $4.7 \%$ ) peuvent alors être rangés en deux groupes aux propriétés complètement différentes:

- Les noyaux proches d' un atome de phosphore, individuellement couplés aux électrons, sont soumis à des interactions hyperfines très différentes les unes des autres, et ne peuvent pas se mettre en équilibre thermique entre eux.

- Les noyaux éloignés d' un atome de phosphore pour lesquels le concept de température de spin s'applique; les échanges d'énergie avec le réseau se font alors par diffusion de spin. Il existe une barrière de diffusion produite par le voisinage d' un atome de phosphore.

\section{Polarisation Dynamique}

La polarisation dynamique fournit d'importantes précisions sur la nature de l'hamiltonien responsable de la relaxation. L' augmentation extrême $A_{\mathbf{m a x}}$ de l'aimantation nucléaire que produit l' effet Overhauser est donnée par $-\gamma_{e} / \gamma_{n}$ pour un couplage scalaire et $+(1 / 2) \gamma_{e} / \gamma_{n}$ pour un couplage dipolaire entre le noyau considéré et un centre paramagnétique [16], et l'on $a$, pour une interaction scalaire,

- D'aprés les mesures de J.C. Irvin, 1960, Communication particulière.

$\dagger$ Echantillon de silicium fourni par Merck. 
La figure 1 présente les résúltats des mesures de l'augmentation par effect overhauser effectuées dans un champ de $3400 \mathrm{G}$. On voit que les valeurs de $1 / A$ s' alignent correctement sur une droite passant par $\left(1 / \Delta_{\max }\right)=(+1 / 3300)$.

On a, pour ${ }^{29} \mathrm{Si}: \gamma_{e} / \gamma_{n}=-3310$ et $l^{\prime}$ on peut conclure que l'interaction est essentiellement scalaire. On conçoit bien que l'interaction de contact soit dominante pour les noyaux proches de l'impureté et que ceux-ci soient polarisés les premiers. Ensuite, l'aimantation nucléaire est transportée à tous les noyaux par diffusion de spin. Ce processus l'emporte sur le couplage dipolaire direct entre les noyaux éloignés et l'atome de phosphore. Ce point de vue sera repris et justifié au chapitre IV.

\section{Relaxation des Noyaux au Voisinage d'une Impureté}

Un centre de phosphore étant choisi comme origine des coordonnées, appelons $\psi(r)$ une fonction d'onde à un électron supposée isotrope, * et dont l'énergie soit voisine de l'énergie de Fermi dans la bande d'impuretés. $\psi(r)$ est normalisée dans le volume $V$ du cristal.

Nous poserons dans la suite $V=1$.

Nous appelons $\Gamma(r)$ le nombre de noyaux de ${ }^{29}$ Si placés dans des sites équivalents à la distance $r$ de l'impureté. Ils forment une classe $\{r\}$ et "voient" la même densité électronique $|\psi(r)|^{2}$.

L' interaction hyperfine de contact entre les spins des électrons et ceux des noyaux de la classe $\{r\}$ s'écrit [16]

$$
\hbar \mathscr{H}=-\frac{8 \pi}{3} \gamma_{e} \gamma_{n} \hbar^{2} \delta(r) \vec{I} \cdot \vec{S}
$$

$\gamma_{e}$ et $\gamma_{n}$ étant les rapports gyromagnétiques des électrons et des noyaux et $\delta(r)$ une fonction de Dirac centrée sur un noyau de $\{r\}$.

Lorsque le système est placé dans un champ magnétique $H, l$ l'interaction $\hbar \mathscr{H}$ perturbe les fréquences de Larmor des noyaux et des électrons; cependant, seules les valeurs moyennes $\left\langle I_{z}\right\rangle$ et $\left\langle S_{z}\right\rangle$ interviennent dans le calcul des niveaux car, d'une part, la fonction d'onde de chaque électron s'étend sur un grand nombre de noyaux et, d'autre part, le spectre des fluctuations dues au mouvement des électrons s'étend à des énergies plus élevées que l'interaction elle-même; ce dernier point est suffisamment établi par la disparition de la structure hyperfine du phosphore pour les concentrations élevées.

Il en résulte pour les noyaux de la classe $\{r\}$ un déplacement de la fréquence de résonance exprimé par

$$
\left.\Delta v\right|_{\{r\}}=\frac{4}{3} \gamma_{e} \gamma_{n} \hbar N|\psi(r)|^{2}<S_{z}>
$$

* En réalité, $\psi(r)$ n'a aucune raison d'être isotrope. Cette hypothèse n'est pas restrictive mais simplifie la discussion, la généralisation à d'autres situations ne pose pas de problème difficile. 
et lorsque le système est à l'équilibre thermique

$$
\left.\frac{\Delta v}{\nu_{0}}\right|_{\{r\}}=\frac{8 \pi}{3}|\psi(r)|^{2} X_{p}
$$

$X_{p}$ est la partie de la susceptibilité statique dépendant du spin par unité de volume et vaut:

$$
x_{p}=N \frac{g \beta S_{0}}{H_{0}}
$$

Cet effet correspond au déplacement de knight dans les métaux [16]. Les noyaux de la classe $\{r\}$ peuvent être identifiés par leur déplacement de knight au moyen des relations (6) ou (7), à condition de connaitre $\left\langle S_{z}\right\rangle$, mais surtout par leur temps de relaxation $T_{1}$.

A basse température et pour une bande large par rapport à $g \beta H_{0}$, la cadence de relaxation caractéristique de la classe $\{r\}$ est donnée par

$$
\left.\frac{1}{T_{1}}\right|_{\{\Delta v\}}=\frac{64 \pi^{3}}{9} \gamma_{e}{ }^{2} \gamma_{n}{ }^{2} \hbar^{3}|\psi(r)|{ }^{4} P_{F}{ }^{2} k T
$$

où $P_{F}$ est la densité d'états au niveaú de Fermi.

Le temps de relaxation et le déplacement de la fréquence des noyaux de la classe $\{r\}$ peuvent être associés par la relation de Korringa

$$
T_{1}\left(\frac{\Delta v}{v_{0}}\right)^{2}=\frac{1}{\pi k T}\left(\frac{N}{\rho_{F}}\right)^{2} \frac{<S_{z}>2}{\gamma_{n}{ }^{2} \hbar H^{2}} .
$$

Par ailleurs, l' aimantation nucléaire $\left\langle I_{z}\right\rangle$ produit un déplacement de la fréquence de Larmor des électrons que l'on peut attribuer à un champ magnétique $H_{n}$ donné par

$$
\left.H_{n}\right|_{\{r\}}=\frac{8 \pi}{3}|\psi(r)|^{2} \gamma_{n} \hbar \Gamma_{\{r\}}<I_{z}>\{r\}
$$

où $l^{\prime}$ indice $\{r\}$ indique qu' il s'agit de la contribution des noyaux de la classe $\{r\}$ au champ $H_{n}$.

Cette dernière propriété a été exploitée pour mettre en évidence la relation de Korringa (10) de la manière suivante $[17,18]$.

Un spectromètre de résonance électronique fonctionnant vers $9400 \mathrm{Mc} / \mathrm{s}$ est réglé au centre de la raie de résonance du phosphore. Il mesure la dérivée de la courbe d'absorption avec un temps de réponse voisin de $0.1 \mathrm{msec}$. La puissance est suffisante pour produire, à $1.5^{\circ} \mathrm{K}$, une saturation de l'ordre de

$$
s=S_{0}-\frac{<S_{z}>}{S_{0}} \approx 0.3
$$


et la polarisation des noyaux de ${ }^{29} \mathrm{Si}$ qui en résulte produit, par l'intermédiaire de la relation (11), un déplacement de la résonance électronique de $0.5 \mathrm{G}$ environ.

Si l' on applique aux noyaux de la classe $\{r\}$ une impulsion de fréquence $v_{0}\left(1+\frac{\Delta v}{v_{0}}\right)$, de durée $\tau$ et d'intensité $h_{1}$ telle que

$$
\gamma_{n} h_{1} \tau=\pi
$$

on renverse l'aimantation de ces noyaux et il en résulte un déplacement de la résonance électronique; le système retourne ensuite à l' équilibre en un temps $T_{1}\{r\}$ dû au temps de relaxation des noyaux suivant (9).

La tension émanant du spectrographe, proportionnelle au déplacement de la fréquence de résonance lorsque celui-ci est petit par rapport à la largeur de raie, donne l'image exacte de $l^{\prime}$ évolution de $\left\langle I_{z}\right\rangle$ pour la classe $\{r\}$. La figure 2 présente quelques exemples.

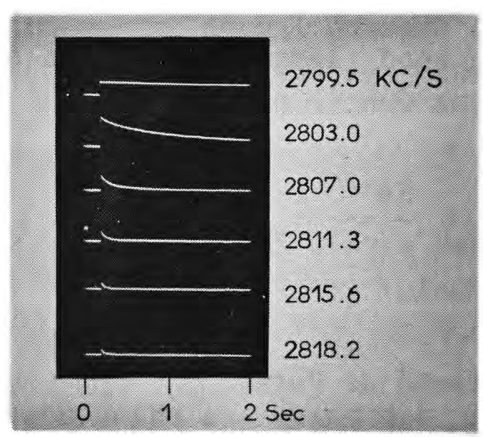

FIGURE 2

Relaxation des noyaux voisins des impuretés qui possèdent des déplacements de Knight différents.

En déterminant ainsi les temps de relaxation associés à toutes les fréquences $v_{0}\left(1+\frac{\Delta v}{v_{0}}\right)$ pour lesquelles un signal est visible on peut, comme le montre la figure 3 , prouver que la relation (10) $S^{\prime}$ applique à toutes les classes $\{r\}$. Dans la figure 3 , on a porté $\left(1 / T_{1}\right)^{1 / 2}$ en fonction de $v$. Les points s'alignent sur une droite.

En opérant à deux puissances différentes (une puissance arbitraire et la moitié de cette puissance), on peut encore déterminer la pente qu' auraient ces droites pour une puissance nulle, c' est-à-dire lorsque l' équilibre thermique est réalisé et que $\left\langle S_{z}\right\rangle=S_{0}$ peut être connu. De ces dernières droites, on tire deux indications:

(1) les temps de relaxation les plus courts que l'on puisse observer (et qui ne sont peutêtre pas les plus courts qui existent et par conséquent ne correspondent pas nécessairement aux premiers voisins) peuvent être associés à un déplacement de Knight de $35 \mathrm{kc} / \mathrm{s}$. Introduisant cette valeur conjointement à $\left\langle S_{z}\right\rangle=0.0085 \pm 0.0010$ mesurée directement (voir chapitre $v$ ) dans la relation (6), on trouve pour la densité électronique au voisinage de l'atome de phosphore dans le silicium conducteur

$$
<\psi(r)>2=0.32 \times 10^{23} \mathrm{~cm}^{-3}
$$

La grandeur correspondante que $l^{\prime}$ on peut extraire des fréquences de transitions ENDOR $v_{I}=2980 \mathrm{kc} / \mathrm{s}$ du phosphore avec ses premiers voisins mesurée par Feher [14] dans le silicium 


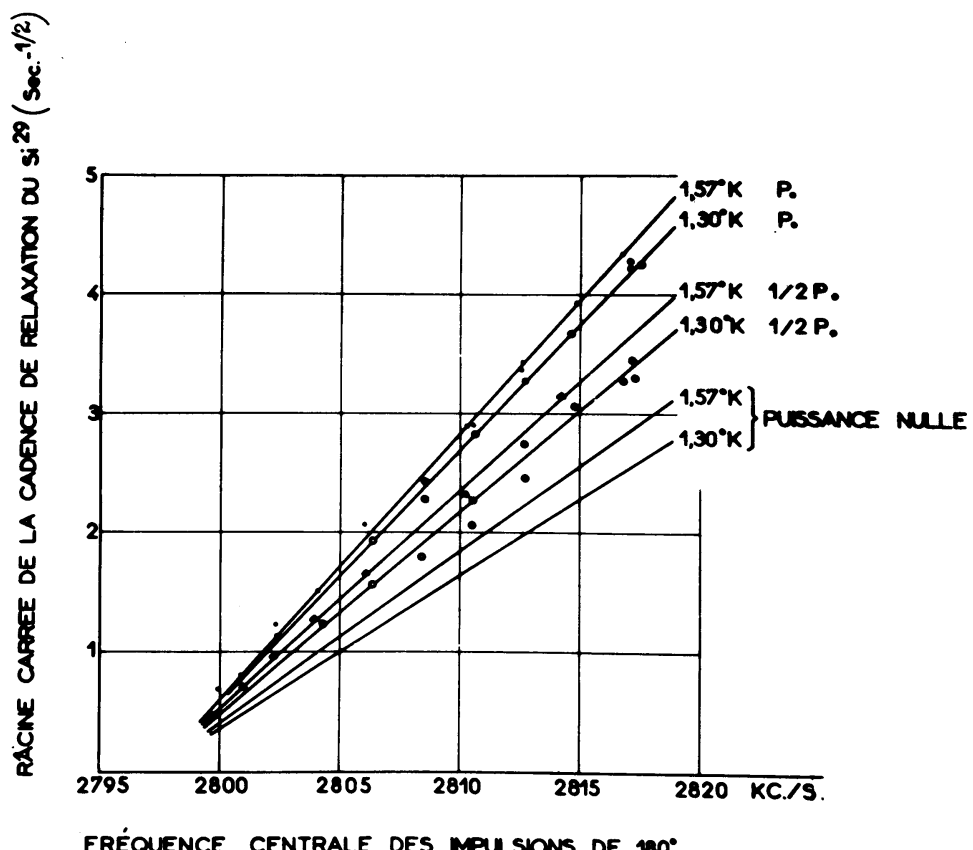

FIGURE 3

Mise en évidence de la relation $T_{1}{ }^{1 / 2}\left(\Delta v / \nu_{0}\right)$ qui est vérifiée pour les noyaux porches des impuretés. Les résultats sont extrapolés pour des polarisations électroniques à $l^{\prime}$ équilibre thermique à $1.30^{\circ} \mathrm{K}$ et $1.57^{\circ} \mathrm{K}$.

non conducteur vaut

$$
|\psi|^{2}=0.44 \times 10^{23} \mathrm{~cm}^{-3} .
$$

Ou voit donc que la densité électronique au voisinage du donneur ne varie pas beaucoup entre des concentrations du phosphore faibles, où le silicium est isolant, et les concentrations élevées, où il est conducteur, et l'on a:

$$
\langle\psi\rangle_{\text {conducteur }}^{2} /|\psi|_{\text {isolant }}^{2} \geqslant 0.72 \text {. }
$$

(2) Au voisinage de $1.5^{\circ} \mathrm{K}$ le temps de relaxation des noyaux est proportionnel à la précision des mesures près:

$$
\begin{aligned}
& \text { d } 1.3^{\circ} \mathrm{K}\left(T_{1} T\right)^{1 / 2} \Delta v=8.7 \\
& \text { d } 1.57^{\circ} \mathrm{K}\left(T_{1} T\right)^{1 / 2} \Delta v=8.6
\end{aligned}
$$

comme il fallait s'y attendre d'après les relations (9) ou (10).

\section{Relaxation des Noyaux Eloignés d'une Impurete}

Pour une concentration de $2.5 \times 10^{18} \mathrm{P} / \mathrm{cm}^{3}$, le rapport entre le nombre de noyaux extérieurs et intérieurs à une sphère de rayon égal au rayon de Bohr du donneur est sensiblement égal a 45 . 
Ainsi pouvons-nous penser qu' un bon nombre de noyaux sont loin de toute impureté, dans une région où la probabilité de présence de l'électron demeure faible. Le signal de résonance s'observe aisément à basse température, mais, par suite des temps de relaxation extrêmement longs ( $10^{2}$ à $10^{4}$ fois plus longs que dans la région proche de l'impureté), la détection de l'aimantation ne peut être faite qu' en passage rapide adiabatique [16]. En effet, pour ne pas saturer le signal d'absorption, on est force d'utiliser des champs de radio-fréquence tellement faibles que ce signal est inobservable pour des polarisations nucléaires correspondant à l' équilibre thermique. Un avantage du passage rapide est de donner directement un signal proportionnel en grandeur et en signe à l'aimantation nucléaire*, toutefois un très fort champ de radiofréquence est nécessaire. Cette condition impose pratiquement l'emploi d' un spectromètre d bobines croisées que l'on règle sur le mode dispersif. La bobine d'émission est placée à l'extérieur de la cavité fendue et la bobine de réception du signal à l'intérieur. Les signaux de résonance respectifs des électrons et des noyaux peuvent ainsi être observés simultanément dans le même champ magnétique $\left(H_{0}=3400 \mathrm{G}\right)[19,20]$. La longueur des temps de relaxation est telle qu' elle rend possible l'étude de la relaxation dans tous les champs compris entre 0 et $10 \mathrm{kG}$. L' observation est toujours faite au même champ $H_{0}$.

\section{(a) Variation de la relaxation nucléaire avec le champ magnétique}

Quel que soit le champ magnétique, la relaxation suit une loi parfaitement exponentielle. Ceci peut s'expliquer de deux façons: ou bien le système des noyaux se met rapidement en équilibre et l'on peut définir une température de spin; ou bien le temps de relaxation dû aux électrons est uniforme loin des impuretés. Nous montrerons que l'expérience confirme le premier point de vue. La figure 4 montre la variation du temps de relaxation avec le champ appliqué.

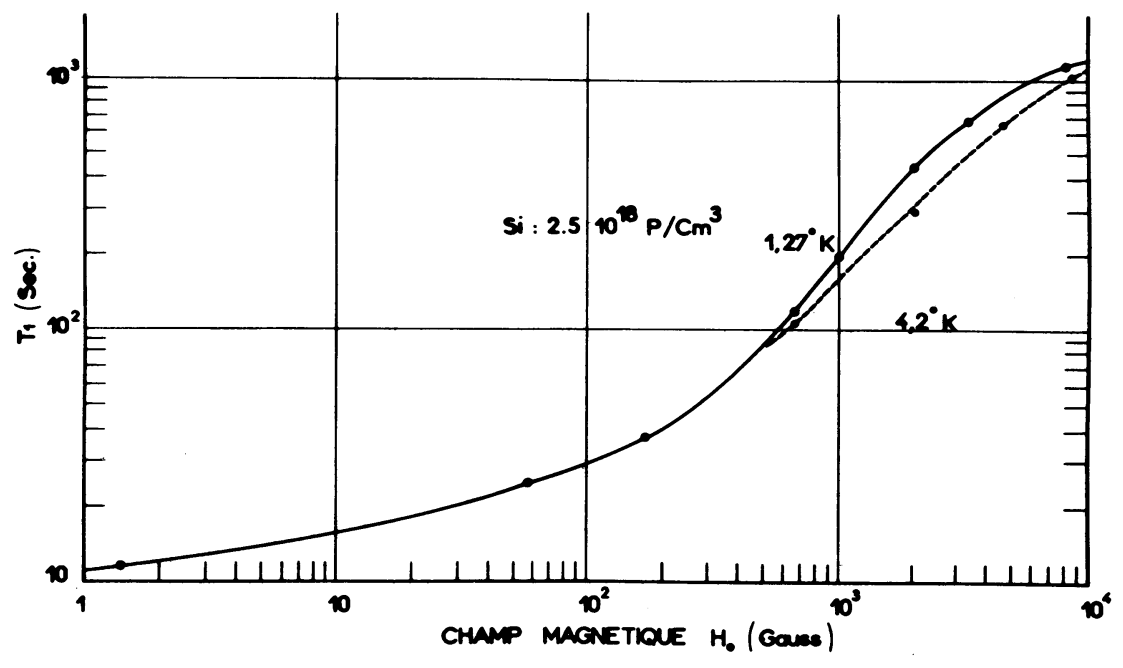

FIGURE 4

Variation du temps de relaxation nucléaire des noyaux éloignés des impuretés en fonction du champ magnétique appliqué.

Pour les électrons dégénérés d'un métal, la relaxation spin-réseau est indépendante du champ appliqué [16] car, en général, les largeưrs de bandes sont très supérieures à l' énergie Zeeman des électrons. Dans le cas de bandes étroites, les densités d’états au niveau de Fermi, $p+F$

* Un gros inceonvénient de cette méthode est de fournir un signal dont la largeur est liée.à l'intensité du champ de radio-fréquence et ne permet pas d'observer la largeur de la raie des spins nucléaires. Nous préciserons, dans la suite, un moyen d'observer cette largeur. 
pour les spins orientés suivant le sens du champ, $P_{-F}$ pour les spins orientés en sens contraire, peuvent être très différentes et le produit $P_{-F}, P_{+F}$ qui intervient dans la relaxation [9] peut alors dépendre fortement du champ appliqué. Cependant nous écarterons par la suite cette explication pour la variation de $T_{1}$ avec le champ magnétique.

\section{(b) Diffusion de spin $[21,22,23]$}

Nous constatons expérimentalement que l'on peut définir avec une très bonne précision une seule constante de relaxation pour les spins éloignés des impuretés, ce qui laisse supposer qu' il y a une diffusion rapide de l'énergie par renversements mutuels de spins proches voisins à partir des régions aù la densité électronique est importante.

Cette diffusion de spin met l'ensemble de tous les spins des régions éloignées des impuretés en équilibre thermique entre eux.

Ces expériences prouvent l'existence de diffusion de spin nucléaire, même en réseau dilué [27]. Nous avons $d^{\prime}$ ailleurs vérifié qu' à $1.3^{\circ} \mathrm{K}$ au bout $d^{\prime}$ un temps de relaxation, le signal de passage adiabatique croît à partir de la valeur nulle, d' une quantité qui est égale à sa croissance à $300^{\circ} \mathrm{K}$ multipliée par le rapport de Boltzmann. A $300^{\circ} \mathrm{K}$, la relaxation nucléaire s' effectue par l'intermédiaire du couplage hyperfin avec les électrons de la bande de conduction, $[16,28]$. La diffusion de spin ne joue donc plus aucun rôle dans la relaxation à la température ordinaire.

En supposant que $l^{\prime}$ on se trouve dans le cas (c) de diffusion rapide de Blumberg [24], nous définissons un rayon $b_{0}$ à partir de l'origine tel que, sur la sphère de rajon $b_{0}$, le déplacement de Knight soit égal au champ local dipolaire $2 \pi \delta / \gamma_{n} \cdot *$

$$
4 / 3 \quad \gamma_{e} \gamma_{n} \hbar N\left|\psi\left(b_{0}\right)\right|^{2}<S_{z}>=\delta \text {. }
$$

A l'extérieur de la barrière, la vitesse avec laquelle l'énergie des spins nucléaires se transmet au réseau est donnée par la formule

- L'observation de la largeur de la raie d'absorption des noyaux ${ }^{29}$ Si dont l'aimantation est augmentée par effet overhauser permet de déterminer 1 'intensité $\delta=109 \mathrm{c} / \mathrm{s}[20]$; voir figure 5 .

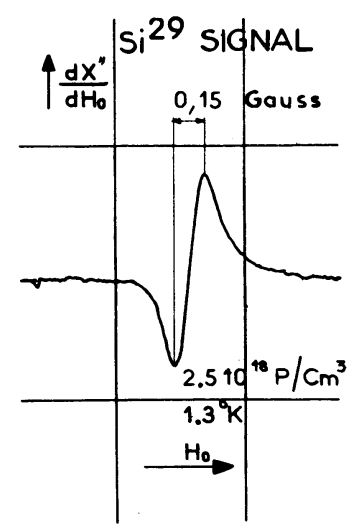

FIGURE 5

Observation de la dérivée du signal d'absorption de ${ }^{29} \mathrm{Si}$ augmenté par effet overhauser à $1.3^{\circ} \mathrm{K}$. 


$$
\frac{1}{T_{1}}=4 \pi N \int_{b_{0}}^{\infty} \frac{r^{2} d r}{T_{1}(r)}
$$

$T_{1}(r)$ est le temps de relaxation qu' aurait un noyau placé à la distance $r$, il est donné par la formule (9).

Dans le cas d' une interaction dipolaire entre les spins nucléaires et un spin électronique localisé, la formule (13) conduit à une variation de $T_{1}$ avec le champ magnétique [24] qui est linéaire. Les résultats de la polarisation dynamique par effect Overhauser (chapitre II) prouvent que le couplage est de nature hyperfine scalaire. Dans (13) la seule variation avec le champ magnétique vient de la borne inférleure d'intégration qui est une fonction monotone et croissante du champ d'après (12). Il en résulte que $T_{1}$ donné par (13) est une fonction croissante du champ magnétique. La dépendance expérimentale de $T_{1}$ avec $H$ (figure 4) a une forme complexe qui se situe entre une dépendance linéaire et une dépendance quadratique

$$
T_{1} \infty H^{\gamma} \quad 1<\gamma<2
$$

Dans l'ignorance où nous sommes de la forme de la fonction d'onde, il est impossible de procéder à une évaluation quantitative de la formule (13).

\section{(c) Barrière de diffusion et temps de polarisation}

L' équation (12) relie l'aimantation électronique moyenne $\left\langle S_{z}\right\rangle$ et le rayon de la barrière de diffusion. La diffusion commence à être efficace d'autant plus près de l'origine que l' aimantation électronique est plus faible. Il y a deux méthodes pour faire varier $\left\langle S_{z}\right\rangle$ : d' une part, à champ constant $H_{0}$ on peut saturer la résonance électronique; d'autre part, les spins électroniques étant en équilibre avec le réseau $\left\langle S_{z}\right\rangle=S_{0}$, on peut faire varier le champ appliqué. Un moyen très simple de mettre en évidence l' existence de la barrière de diffusion consiste à étudier le temps de polarisation par effect overhauser pour divers taux de saturation de la résonance électronique.

L' aimantation nucléaire augmentée par effet overhauser est la solution stationnaire de 1 'équation d'évolution [16].

$$
\frac{d<I_{z}>}{d t}=-\frac{1}{T_{1}}\left\{\left\langle I_{z}>-I_{0}\left(1-s \frac{\gamma_{e}}{\gamma_{n}}\right)\right\}\right. \text {. }
$$

D' après (15), la vitesse de polarisation $1 / T_{p}$ doit être égale à la vitesse de relaxation $1 / T_{1}$. Or, comme le montre la figure 6 , ce n'est que pour une saturation nulle $\left\langle S_{z}\right\rangle=S_{0}$ que la vitesse de polarisation est égale à la vitesse de relaxation, et l'on constate par exemple que, pour un coefficient de saturation $s=0.25$, le temps de polarisation décroit de $30 \%$, ce qui est en relativement bon accord avec la diminution du temps de relaxation produite par une diminution de $25 \%$ du champ magnétique appliqué $H$ (voir figure 4).

En champ bas, la diffusion débute très près de l'impureté dans une région de forte densité électronique où les vitesses de relaxation directes sont importantes. Le palier traduit vraisemblablement la constance de la densité électronique dans le très proche entourage de l'impureté. En champs élevés, la barrière de diffusion se trouve dans une région où le gradient radial de la densité électronique $\langle\psi(r)\rangle$ devient très faible, d' où le nouveau palier. 


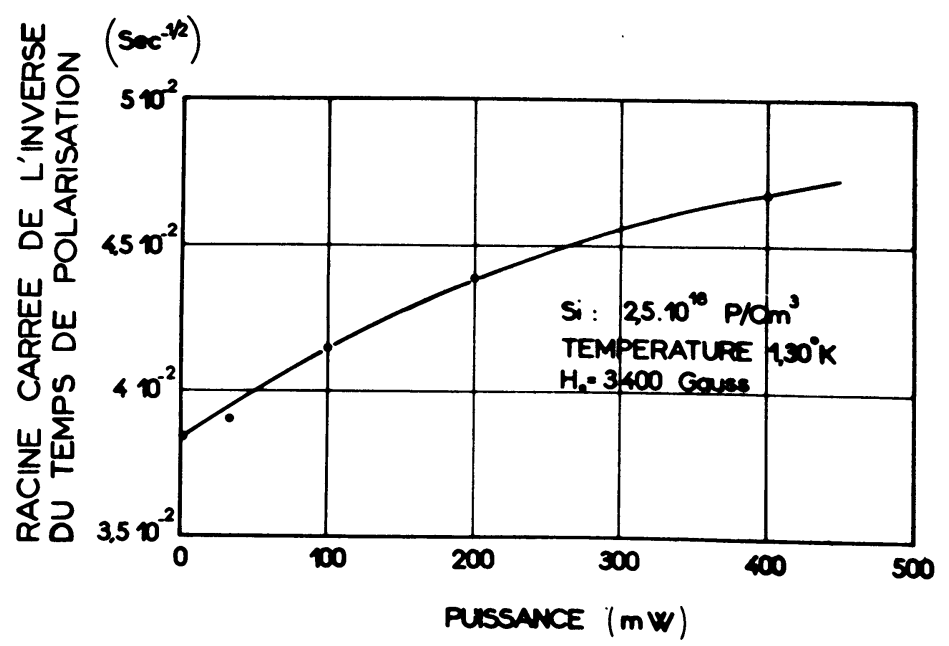

FIGURE 6

Variation de la racine carrée de la vitesse de polarisation produite par effet overhauser en fonction de la puissance de saturation hyperfréquence.

\section{Statistique des Electrons dans la Bande d' Impuretes}

Pour $N$ spins électroniques par unité de volume dans un isolant, c' est-d̀-dire suffisamment découplés les uns des autres, la susceptibilité demeure inversement proportionnelle à la température [29]

$$
x_{p}=N \frac{(g \beta)^{2}}{4 k T}
$$

Pour un gaz d' électrons sans interaction obéissant à une statistique de Fermi, seuls les états dont l'énergie est comprise dans une bande étroite de l'ordre de $k T$, au voisinage du niveau de Fermi, contribuent à la susceptibilité (16) réduite dans le rapport $T / T_{F}$ et devenant par conséquent indépendante de la température. La comparaison de la suscept1bilité des électrons de la bande à celle des électrons d' un sel paramagnétique obéssant à la loi de Curie (16) fournit donc des indications sur le degré de dégénérescence et la forme de la bande. Dans notre cas, le sel paramagnétique de référence est $\mathrm{SO}_{4} \mathrm{Cu}, 5 \mathrm{H}_{2} \mathrm{O}$ monocristallin. L' aire délimitée par les courbes d'absorption observées dans les mêmes conditions est proportionnelle aux susceptibilités statiques. Nous obtenons, pour le rapport de la susceptibilíté des électrons liés à celle des électrons de la bande, par comparaison des deux signaux de résonance observés simultanément,

$$
\begin{array}{ll}
\left.\frac{x_{p}(\text { liés })}{\left.x_{p} \text { (bande }\right)}\right|_{1.24^{\circ} \mathrm{K}}=9.73 & \left.\frac{x_{p} \text { (liés) }}{\left.x_{p} \text { (bande }\right)}\right|_{4.20^{\circ} \mathrm{K}}=4.37 \\
\frac{x_{p}(\text { liés })}{\left.x_{p} \text { (bande }\right)} T=12.6 & \frac{x_{p}(\text { liés })}{\left.x_{p} \text { (bande) }\right)} T=18.5
\end{array}
$$


La susceptibilité du sel paramagnétique suit la loi (16). De ces résultats, on détermine la variation de la susceptibilité de la bande avec la température

$$
\frac{X_{p}\left(4.20^{\circ}\right)}{X_{p}\left(1.24^{\circ}\right)}=0.685
$$

Pour les électrons complètement dégénérés d' une bande large dans un métal, ce rapport serait constant. La valeur moyenne de l'aimantation $\left\langle S_{z}\right\rangle=\frac{h v_{e}}{4 k T} \frac{X_{p} \text { (bande) }}{X_{p}(\text { liés })}$ s'obtient aisément à partin de (17).

$A 1.24^{\circ} \mathrm{K}$,

$$
\left\langle S_{z}>=0.0085\right.
$$

Les résultats (18) font ressortir une variation de $30 \%$ de la susceptibilité entre $1.24^{\circ} \mathrm{K}$ et $4.20^{\circ} \mathrm{K}$. Cela pourrait sembler incompatible avec une susceptibilité déjà réduite à $4.2^{\circ} \mathrm{K}$ par un facteur 4.37 (16), donc susceptibilité d'un gaz déjà dégénéré. Cependant, à basse température, il est possible d'obtenir une valeur approchée de $x_{p}$ faisant intervenir la courbure au niveau de Fermi de la bande représentant la densité d'états [29] (figure 7):

$$
x_{p}(T)=x_{p}(0)\left(1+\frac{\pi^{2}}{6}(k T)^{2} \frac{\rho_{F}^{\prime \prime}}{\rho_{F}}\right) .
$$

Le second terme peut prendre une valeur négative importante si la courbure de la bande est forte, c'est-à-dire lorsque le niveau de Fermi se trouve sensiblement au milieu d' une bande

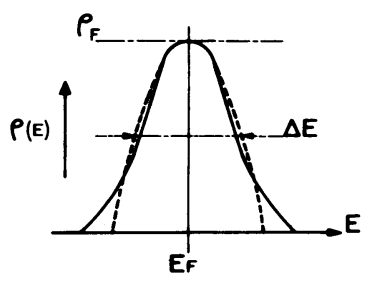

FIGURE 7

Représentation schématique de la densité d'états de la bande d'impuretés.

La parabole osculatrice qui permet de définir la largeur $\Delta E$ a été représentée en traits ponctués.

très étroite (fugre 7). Dans une estimation de la largeur de la bande, nous admettons que la densité d'états s'assimile à une parabole au voisinage du niveau de Fermi.* La largeur de la

* Des calculs ont été faits pour obtenir la structure de la bande en fonction de la concentration des impuretés supposées situées aux noeuds d'un réseau périodique. Toutefois, pour une distribution au hasard, on peut montrer $[11,12,13]$ que 1 a bande, tout en continuant à présenter un maximum étroit, se prolonge par des queues vers les hautes et basses énergies. C'est une forme justifiée par des modèles exacts à une dimension. 
parabole à mi-hauteur $\Delta E$ peut $s^{\prime}$ obtenir à partir de (18) et (20). On trouve $\Delta E=18.6^{\circ} \mathrm{K}$.

Nous arrivons à la conclusion que les électrons, vraisemblablement très dégénérés dans la bande à $1.24^{\circ} \mathrm{K}$, le demeurent à $4.2^{\circ} \mathrm{K}$. Effectivement, les mesures de relaxation nucléaire en fonction de la température montrent une vitesse de relaxation proportionnelle à la température à $1.24^{\circ} \mathrm{K}(9)$. Une telle loi de variation se rencontre lorsque les électrons suivent une statistique de Fermi sans avoir à formuler d'hypothèse sur la forme de la bande. Pour des températures supérieures à $4.2^{\circ} \mathrm{K}$, la vitesse de relaxation varie plus lentement avec la température. Les mesures [30] effectuées à $10,000 \mathrm{G}$ le prouvent aussi.

En résumé, la largeur de bande déduite de sa courbure au niveau de Fermi est $\infty 19^{\circ} \mathrm{K}$. Il est difficile de préciser cette largeur et notamment une énergie de Fermi, faute d' une bonne connaissance de la forme de la bande.

\section{Remerciements}

Nous remercions Monsieur le Professeur Abragam qui nous a suggéré une partie de ces expériences, et dont les conseils nous ont èté précieux. Nous remercions aussi Monsieur M. Goldman pour d'utiles discussions.

\section{References}

1. F.J. MORIN et J.P. MAITA, Phys. Rev, 96, 28 (1954).

2. R.0. CARLSON, Phys. Rev. 100, 1075 (1955).

3. H. FRITZSCHE et M. CUEVAS, Phys, Rev, 119, 1238 (1960).

4. E.C. McIRvine, J. Phys. Chem. Solids 15, 356 (1960).

5. N.F. MOTT, Canad. J. Phys. 34, 1356 (1956).

6. N.F. MOTT, Phil. Mag. 6, 287 (1961).

7. E. WIGNER, Trans. Faraday Soc. 34, 678 (1938).

8. W. KOHN, Phys. Rev. 133A, 171 (1964).

9. N.F. MOTT et W.D. TWOSE, Adv. Phys, 10, 107 (1961).

10. R. BOWERS, Phys. Rev. 108, 683 (1957).

11. P. AIGRAIN, Physica 20, 978 (1954).

12. J. DESCLOIZEAUX, J.Phys. Rad. 20, 606 (1959).

13. E.M. CONWELl, Phys. Rev. 51, 103 (1960).

14. G. FEHER, Phys. Rev. 114, 1219 (1959).

15. D. JEROME et J.M. WINTER, Phys, Rev. 134A, 1001 (1964). 
16. A. ABRAGAM, Principles of Nuclear Magnetism, Oxford (1961).

17. I. SOLOMON, C.R. Colloque Ampère, North-Holland, Eindhoven (1962).

18. Ch. RYTER, Phys. Rev. Letters 5, 10 (1960).

19. J. COMBRISSON et I. SOLOMON, J. Phys. Rad. 20, 683 (1959).

20. D. JEROME, Thèse d'état, Université d'orsay, à paraitre.

21. N. BLOEMBERGEN, Physica 15, 386 (1949).

22. G.R. KHUTSISHVILI, Soviet Phys. J.E.T.P. 15, 909 (1962).

23. P.G. DE GENNES, J. Phys. Chem. Solids. 7, 345 (1958).

24. T.E. BLUMBERG, Phys. Rev. 119, 79 (1960).

25. W. KOHN et J.M. LUTTINGER, Phys. Rev. 97, 869 (1955).

26. W. KOHN, Solid State Phys. 7 (édité par F. Seitz et D. Turnbull), Academic Press, New-York (1957).

27. P. W. ANDERSON, Phys. Rev. 109, 1492 (1958.

28. G. LAMPEL et I. SOLOMON, C.R. Acad. Si. 258, 2289 (1964).

29. N.F. MOTT et H. JONES, Theory of the properties of alloys, Oxford (1936).

30. R. K. SUNDFORS et D.F. HOLCOMB, Phys. Rev. 136A, 810 (1964). 